\title{
Proceso de atención de enfermería integral emergente del paciente politraumatizado en el área de emergencia del hospital Luis Fernando Martínez Amoroso de la ciudad de Cañar
}

\section{Process of comprehensive nursing care of the polytraumatized patient in the emergency area of the hospital Luis Fernando Martínez Amoroso of the city of Cañar}

\author{
Bustamante Espinoza Luisa Katerine \\ Carrera de Enfermería,Universidad Católica de Cuenca \\ Cañar,030201,Ecuador \\ lkbustamantee@ucacue.edu.ec
}

\begin{abstract}
Resumen
Proceso de atención de enfermería integral emergente del paciente politraumatizado en el área de emergencia del hospital Luis Fernando Martínez Amoroso de Cañar, cuyo objetivo del trabajo es Diseñar un sistema de valoración emergente, para garantizar la atención del paciente con politrauma, con la metodología de investigación de campo, con paradigmas cualitativos y cuantitativos ,con la aplicación de una guía de observación y encuestas, con una población de 19 enfermeras, 25 auxiliares de enfermería y 12 internas de enfermería, se encuentra sin una correcta aplicación del proceso de atención de enfermería basada en las taxonomías NANDA NIC Y NOC, por lo que se ve la necesidad de diseñar un sistema de valoración emergente para garantizar el bienestar del paciente con politraumas.
\end{abstract}

Palabras clave: Proceso de atención de enfermería, taxonomías, politraumatizado, trauma.

\begin{abstract}
Integral Emerging-Comprehensive process of nursing acting to politraumatized patients at the emergency room of the Luis $F$ Martinez hospital in Cañar city that pretends to design an urgent assessment system in order to guarantee the quality of nursing attention and care for politraumatized patients, using research field methodology and taking into account quality and quantity paradigms in which questionnaires and an observational guide was applied, a sample of 19 nurses, 12 internal nursing residents, and 25 auxiliary nurses was taken into study, in whom a deficient assessment nursing care was found, as well as an inadequate application of the nursing attention process based on the NANDA NIC and NOC taxonomies. Due to these factors, it is extremely necessary the design of an urgent assessment system which guarantees the wellbeing of a politraumatized patient
\end{abstract}

Key words: Nursing process, taxonomies, multiple trauma, trauma.

\section{Introduccion}

El objetivo de la investigación es Diseñar un proceso de atención de enfermería integral emergente, para garantizar la atención del paciente politraumatizado, no existe dicho proceso en el área de emergencia del Hospital Luís Fernando Martínez Amoroso.

En el mundo el politraumatismo es una de las primeras causas de muerte en personas productivas, trauma es definido como "una lesión o daño que puede causar la muerte a nivel orgánico, resultado de la exposición aguda a un tipo de, energía térmica, eléctrica, energía mecánica radiante o química, en conjuntos que superan el umbral de la tolerancia fisiológica. ${ }^{1}$.

Se considera el trauma como un problema de salud pública. La mortalidad es el indicador principal magnitud del problema de salud pública, teniendo en consideración que por cada sobreviviente suele existir secuelas secuelas emocionales y físicas. Afectando a las familias de la víctima, comunidades y a la sociedad. Los gastos ocasionados por trauma llegan a ser grandes y se miden en la estimación de los años de vida perdidos (AVPP) y los años de vida 
ajustados por discapacidad (AVISA). Los AVPP se calculan, la diferencia entre la edad promedio de supervivencia en la sociedad y la edad de la víctima ${ }^{2}$; los AVISA se estiman mediante la ponderación de estos años perdidos teniendo en cuenta la causa, la incapacidad y las edades en que la muerte se da. ${ }^{3}$ Se estima que en el año 2000 mas o menos unos cinco millones de personas tuvieron un desenlace fatal como resultado de un trauma. ${ }^{3}$ La mayoría de muertes producidas por trauma ocurren en los países de bajo y mediano ingreso según la clasificación del banco mundial (The World Bank, 1998) y ocurre a personas jóvenes, entre los 15 y 44 años de edad, la etapa económica más productiva en la vida de una persona. La mayoría de los traumatismos son más prevalentes en hombres que en mujeres. ${ }^{3}$.

En el Hospital Luís Fernando Martínez Amoroso, de la ciudad de Cañar en el área de emergencia tenemos que el personal de enfermería es el primer miembro del equipo de salud que recibe al paciente cuando ingresa al hospital tiempo valioso para la victima de traume, en el que depende su vida, la enfermera actúa hasta que el médico llegue, la enfermera no enfatiza sus criterios técnico científico para reaccionar y actuar de emergente, y de esta manera satisfacer las necesidades que el paciente luego del trauma presente El paciente con politrauma en coma o, no puede expresar su el dolor o sufrimiento, y que podríamos encontrar deterioro del estado general del paciente por daños o respuestas de sistemas del organismo a nivel endócrino, metabólico que ayudan a perjudicar el estado general del paciente donde experimenta cambios neurológicos son un proceso neurológico y fisiológicos, en ese momento al tardar con las actuaciones de enfermería, el paciente experimenta complicaciones, no inmovilización adecuada dependiendo el caso, va experimentar más dolor, lo que conlleva a ansiedad, irritabilidad, aumenta el sufrimiento, impactando a sus familiares. Manejo desordenado nos hace perder tiempo en el accionar de enfermería, la falta de aplicación de conocimientos, sobre el triage en casos de accidentes, no se distribuye, las actividades de forma adecuada, ya que se en caso de accidentes de tránsito donde existe varias víctimas se aglutina personal sin asignación de roles y actividades específicas. ${ }^{2}$.

De seguir subsistiendo el problema y la enfermería mantenerse debilitada, afecta la salud del paciente, tanto física como psicológica.

\section{Materiales y Métodos}

- Observación de los fenómenos directamente para poder identificar el problema.

- Concepción de opiniones y evaluación de vicisitudes y fenómenos.

- Evaluación de la posibilidad de que la idea a defender se materialice y concrete en la práctica.
Tabla 1. Grafo que representa la red física de un ISP.

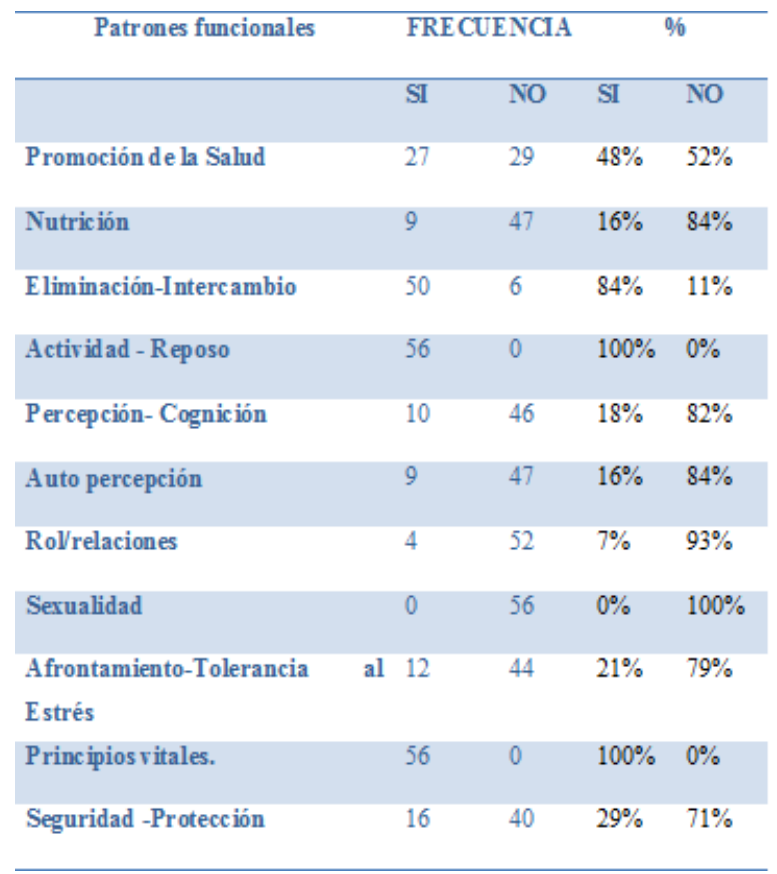

Elaborado por: Luisa Katerine Bustamante Espinoza

- Generación de doctrinas como proceso de creación teórico- práctica.

- Las técnicas más utilizadas es la observación y la encuesta y sus instrumentos correspondientes.

Los datos de la tabla 1 nos dan a conocer los parámetros más valorados cuando se recepta al paciente en el área de emergencia son: 48 \% Nutrición, 84 \% EliminaciónIntercambio, $100 \%$ Actividad - Reposo, $100 \%$ con un Principios vitales y $100 \%$, Confort, entendiendo que el accionar de enfermería se retorna rutinarias, no existe valoración de patrones funcionales basadas en las taxonomía NANDA, NIC; NOC.

Con los datos recolectados en la tabla 2 se evidenció que el paciente politraumatizado que es recibido en emergencia es sometido a una serie de procedimientos en el que el personal de enfermería consta como miembro activo del equipo de salud, procedimientos en el que se debe asiste al médico como son: $66 \%$, intubación endotraquial $77 \%$. Canaliza vena de calibre grueso. $100 \%$ Controla de volemia mediante la administración de líquidos endovenosos. $80 \%$ aspiración de secreciones de acuerdo con las necesidades del paciente. $86 \%$ realiza curación de heridas y mantiene apósitos secos con lo que vigila el sangrado. 59\% Inmoviliza las articulaciones con lesiones agudas, el personal de enfermería realiza sus procedimientos en forma de rutina, deja las actividades que las haga los internos de medicina y no se desenvuelven en procedimientos más importantes ya sea por falta de práctica, por poco conocimiento científico, 
Tabla 2. ¿Qué procedimientos se debe realizar a un paciente que ingresa a emergencia? OPCIONES. FRECUENCIA PORCENTA.J

\begin{tabular}{|c|c|c|c|c|}
\hline & SI & NO & SI & NO \\
\hline $\begin{array}{l}\text { ¿despeja vias área mediante elevación del mentón por tensión de la mandibula, sin } \\
\text { hiperextensión del cuello? }\end{array}$ & 4 & 52 & $7 \%$ & $93 \%$ \\
\hline ¿Administra $\mathrm{O}_{2}$ a concentraciones al tas mediante dispositivos seguinla necesidad del paciente? & 56 & 0 & $100 \%$ & $0 \%$ \\
\hline ¿Ayuda al médico durante la intub ación endotraquial? & 37 & 19 & $66 \%$ & $34 \%$ \\
\hline ¿Calienta a temperahura adecuadalas solvciones cristaloides? & 56 & 0 & $100 \%$ & $0 \%$ \\
\hline ¿Canaliza vena periférica de calibre grueso? & 43 & 13 & $77 \%$ & $23 \%$ \\
\hline $\begin{array}{l}\text { ¿En caso de hemorragia activa aplica presión directa sobre el sitio sangrante con un vendaje } \\
\text { compresivo o una compresa estéril? }\end{array}$ & 14 & 42 & $25 \%$ & $75 \%$ \\
\hline ¿Valora signos de taponamiento cardiaco? & 21 & 35 & $37 \%$ & $63 \%$ \\
\hline ¿Monitoriza permanentemente signos vitales? & 56 & 0 & $100 \%$ & $0 \%$ \\
\hline ¿Controla de volemia del paciente mediante la administración de liquidos endovenos os?. & 56 & 0 & $100 \%$ & $0 \%$ \\
\hline ¿Ejecuta aspiración de secreciones de acuerdo con las necesidades del paciente? & 45 & 11 & $80 \%$ & $20 \%$ \\
\hline ¿Trata el dolor con administración de analgésicos, según prescripción médica? & 56 & 0 & $100 \%$ & $0 \%$ \\
\hline $\begin{array}{l}\text { ¿Realiza curación de heridas y mantiene apósitos secos con lo que } \\
\text { vigila el sangrado?. }\end{array}$ & 48 & 8 & $86 \%$ & $14 \%$ \\
\hline ¿Inmoviliza las articulaciones con lesiones agudas? & 33 & 23 & $59 \%$ & $41 \%$ \\
\hline
\end{tabular}

Elaborado por: Luisa Katerine Bustamante Espinoza

por comodidad o solo aborda la parte administrativa llenado de formularios, descuidando el cuidado directo.

\section{Discusión}

Al terminar la investigación se concluye que el personal de enfermería no recibía capacitación adecuada para la atención de los pacientes politraumatizados que acudían al área de emergencia, esto se vio reflejado en las encuestas realizadas en el personal de enfermería que labora en el área de emergencia del Hospital Luís Fernando Martínez.

Obteniendo los datos de la encuesta indica que le mayor porcentaje de los patrones funcionales que se valora en emergencia son: nutrición el $52 \%$, Eliminación/Intercambio $16 \%$, actividad-reposo $100 \%$, principios vitales con un $0 \%$ y confort $0 \%$ el proceso de valoración no continua poniendo en peligro la vida, de ahí la importancia en la etapa de valoración.

El personal de enfermería no realiza una valoración neorológica, poniendo más interés en los procedimientos rutinarios y quitando la importancia de una valoración neorológica.

El personal de enfermería no emite su diagnóstico teniendo un $43 \%$, lo que indica que no conoce el estado real del paciente politraumatizado por lo que no podrá planificar los cuidados integrales oportunos y seguros.

Un $70 \%$, se preocupa en preservar la vida, $23 \%$ se encarga solo con cumplir la prescripciones médicas, el $7 \%$ se ocupa del accionar propio de enfermería eso nos refiere que no existe toma de decisiones para el cuidado del paciente.

\section{Conclusiones}

El accionar en la atención del paciente politraumatizado del personal de enfermería tiene gran valor, pero al no ser cumplida a cabalidad, entorpece la evolución favorable del paciente con politrauma, la estabilización con actuación ágil y segura basada en toma de decisiones con un criterio científico de esta forma evitar complicaciones permanente que puede llegar hasta la muerte del paciente.

No existe un Proceso de Atención de Enfermería protocolizado en pacientes politraumatizado en el área de emergencia los encuestados respondieron el $100 \%$ que no, insistiendo la importancia de implementar Proceso de Atención de Enfermería protocolizado. 


\section{Agradecimientos}

Agradezco al personal de enfermería del hospital "Luis Fernando Martínez Amoroso" y a la Universidad Católica de Cuenca extensión Cañar.

\section{Referencias}

1. Nancy H. Planes de Cuidados en enfermería médicoquirúrgica. Edición Doyma Barcelona. 1988;1.

2. Carpenito LJ. Manual de diagnóstico de enfermería. unirioja, editor. McGraw-Hill Interamericana de España; 1995.

3. of Surgeons Committee on Trauma AC, et al. ATLS, Programa Avanzado de Apoyo Vital en Trauma para Médicos. Comité de Trauma del Colegio Americano de Cirujanos Chicago. 1997;

Recibido: 01 de junio de 2016

Aceptado: 04 de mayo de 20170

Bustamante Luisa: Licenciada en Enfermería, Docente Universitario. 